\title{
Application of Gray Neural Network Combined Model in Transformer Top-oil Temperature Forecasting
}

\author{
Ya Yuan Yla , Ran LI \\ School of Electrical Engineering, South West Jiaotong University, Chengdu 610031, China
}

\begin{abstract}
In order to forecast the transformer top-oil temperature accurately, quickly and efficiently, the paper proposes a prediction model of the transformer top-oil temperature based on Grey Neural Network. Such factors impacting transformer top-oil temperature as load current, ambient, active power and reactive power are comprehensively considered in the prediction of top-oil temperature, and the combined forecasting model which verified the applicability, accuracy and feasibility is established. The example shows that the forecasting results by the proposed method are better than those by BPNN method and the generalization ability of BPNN is improved. The proposed method possesses the following good properties: high precision of forecasting, fast convergence, nice commonality and its average relative error is within the range of $1 \%$.
\end{abstract}

\section{Introduction}

Transformers are the important equipments in the process of power generation and power supply. Having a very high position in the power grid, transformers are the key equipments to ensure network security, reliability, economic operation of power system and people's production and living electricity.

In recent years, with the rapid growth of electricity demand, high-speed construction and investment of power grid drive the market demand of power transmission and transformation equipments. 《Action Plan of Distribution Network Construction and Reconstruction(2015-2020) 》 points out that the investment of distribution network construction will be not less than 2 trillion yuan in 2015-2020. There will be not less than 1.7 trillion yuan in a cumulative investment during the period of "the 13th five-year plan". Therefore, the upgrade and optimization of transformers are the new development trends of future. The high efficiency and energy saving, and the high overload capacity of transformers will be widely used. However, the transformer top-oil temperature is a key factor that affects load capacity, manufacturing cost and service life of the transformer. The transformer top-oil temperature overrun will bring huge damage to the transformer and affect the normal operation of the power grid seriously. There are many strict requirements on transformer top-oil temperature in the specifications. The actual transformer is commonly alarming in $70^{\circ} \mathrm{C} \sim 75^{\circ} \mathrm{C}$, and tripping operation in $80^{\circ} \mathrm{C} \sim 85^{\circ} \mathrm{C}$ and the highest temperature is $95^{\circ} \mathrm{C}$. When the cooling system fails to remove all the coolers, the forced oil cooling transformer and the strong oil water cooling transformer will be allowed to operate
20 min under the rated load. It is allowed up to $75^{\circ} \mathrm{C}$ when the oil surface temperature has not reached $75^{\circ} \mathrm{C}$. But the longest running time will be not more than $1 \mathrm{~h}$ after the removal of the cooler. Thus, the accurate prediction of transformer top-oil temperature has a very important significance on our understanding of the transformer operation and preventing the significant thermal accident.

At present, there are many methods to predict the topoil temperature, such as the indirect measuring method ${ }^{[1-3]}$, the direct calculating method ${ }^{[4-9]}$, the intelligent prediction method ${ }^{[10-12]}$ and so on. Qing $\mathrm{He}^{[13]}$ of Arizona State University established top-oil temperature prediction model by using three different methods: static neural networks, temporal processing networks and recurrent networks. Considering the influencing factors of load current and ambient temperature, the forecast results were compared with those of regression models. The feasibility of prediction model was verified while the prediction accuracy needed to be improved. Chen Weigen $^{[14]}$ of Chongqing University proposed the generalized neural network prediction model which using the environmental temperature, top-oil temperature, bottom temperature, dead end temperature and lower dead temperature as input layer neurons. Experiments were carried out under different loads and different oil flow velocities, and the results show that the prediction method was accurate. Due to the defects of the neural network itself, the model lacked the actual physical meaning. Kalman filtering algorithm was used by Wang Yongqiang $^{[15]}$ to predict the top-oil temperature. Constructing the top-oil temperature state equation and measurement equation based on Kalman filter, the forecasting method was reliable and accurate by the simulation. Because of the hysteresis of the model, the

\footnotetext{
${ }^{\mathrm{a}}$ Corresponding author:319306111@qq.com
} 
forecast results had deviation when the load and the environment temperature changed fast.

The paper establishes a prediction model of transformer top-oil temperature based on Grey Neural Network which considering the transformer load current, environment temperature, active power, reactive power and the top-oil temperature of the previous time. The prediction method is validated by the data collected from a power supply company in Chengdu. The results show that the prediction model of transformer top-oil temperature is accuracy and superior to BP neural network model. The proposed model improves the top-oil temperature prediction accuracy and can be used to instruct the transformer load operation mode and enhances the safety and reliability of the operation of the power grid.

\section{Basic theory of grey neural network}

Considering the different factors influencing transformer top-oil temperature, the top-oil temperature can be regarded as a complex process with large randomness. The grey theory and neural network theory are combined, which can improve the prediction accuracy of the top-oil temperature. The combined model has a good capability of nonlinear mapping and can weaken the randomness of the raw data.

\subsection{Grey theory}

Grey theory which is a new method to study the problem of less data, poor information and uncertainty was proposed by Professor Deng Julong in 1982.

It is a "small sample", "poor information", uncertain system which known to some information and unknown to part of the information as the research object. The proposed model achieves the correct description and effective monitoring of system's function and disciplinarian through the understanding of part known information.

The models of the grey system are called grey models, which are differential equations based on the original data sequence. Assuming that there is a sequence of raw data $x^{(0)}$ :

$$
x^{(0)}=\left(x_{t}^{(0)} \mid t=1,2, \mathrm{~L}, n\right)=\left(x_{1}^{(0)}, x_{2}^{(0)}, \mathrm{L}, x_{n}^{(0)}\right)(1)
$$

$x^{(0)}$ makes a accumulation to get a new data sequence $x^{(1)}$. The item t of $x^{(1)}$ is the sum of the previous $\mathrm{t}$ terms of $x^{(0)}$ and has the form:

$$
x^{(1)}=\left(x_{t}^{(1)} \mid t=1,2, \mathrm{~L}, n\right)
$$

Where

$$
x_{n}^{1}=\sum_{t=1}^{n} x_{t}^{(0)} \text {. }
$$

According to the new data sequence $x^{(1)}$, the whitening equation will be established and is given by:

$$
\frac{d x^{(1)}}{d t}+a x^{(1)}=u
$$

Solving (3) we get,

$$
x_{t}^{*(1)}=\left(x_{1}^{(0)}-u / a\right) e^{-a(t-1)}+u / a
$$

$x_{t}^{*(1)}$ is the estimate of $x_{t}^{(1)}$. Made a regressive, $x_{t}^{*(1)}$ gets the predictive value $x_{t}^{*(0)}$ of $x^{(0)}$ and is given by:

$$
x_{t}^{*(0)}=x_{t}^{*(1)}-x_{t-1}^{*(1)} \quad t=2,3, \mathrm{~L} n
$$

\subsection{Gray neural network}

According to the analysis of grey theory, $x_{t}^{(0)}$ $(t=0,1,2, \cdots, N-1)$ makes a accumulation to get a new data sequence $x_{t}^{(1)}(t=0,1,2, \cdots, N-1)$ which is in accordance with the law of some kind of functions. Described by a continuous function or differential equation, the new data sequence can be predicted by data fitting.

Through the above analysis, the differential equation of the grey neural network can be expressed as follows

$$
\left\{\begin{array}{l}
\frac{d y_{1}}{d t}+a y_{1}=m \\
m=b_{1} y_{2}+b_{2} y_{3}+\mathrm{L}+b_{n-1} y_{n}
\end{array}\right.
$$

In the formula, $y(t)$ represents the $x_{t}^{(1)}$ above; $y_{i}(i=2, \cdots, \mathrm{n})$ is a input parameter for the system; $y_{1}$ is the output parameter for the system; $a, b_{\mathrm{j}}(j=1$, $2, \cdots, n)$ are the coefficients of the differential equation.

The time response equations of (6) are

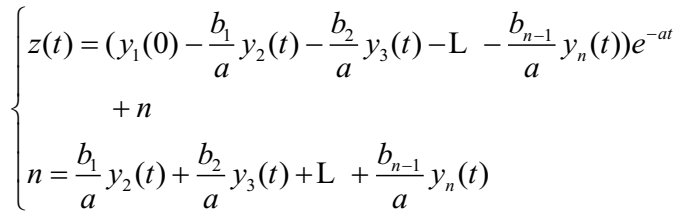

Mapping to an extended BP neural network, the transformed formula (7) will be the gray neural network which has $\mathrm{n}$ input parameters and 1 output parameter. The topology of the network is shown in Figure 1.

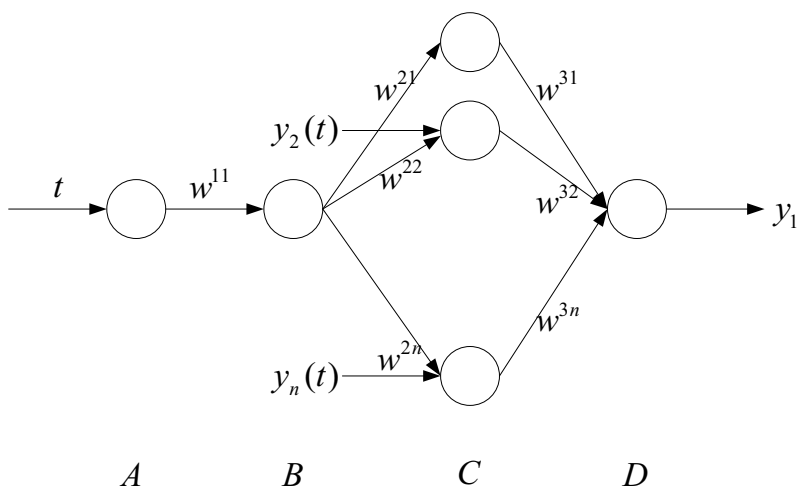

Figure 1 The topology of the network

In the figure $1, t$ is the serial number of the input parameters; $y_{i}(i=2, \cdots, n)$ is the input parameter of the network; $w^{l k}(l=2,3 ; k=1,2, \cdots, n)$ is the weight of the network; $y_{1}$ is the prediction value of the network; $A$ 、 $B 、 C 、 D$ are respectively represented four layers of gray neural network.

\subsection{Gray neural network learning process}

Through the above analysis, the gray neural network learning process is shown in Figure 2. 


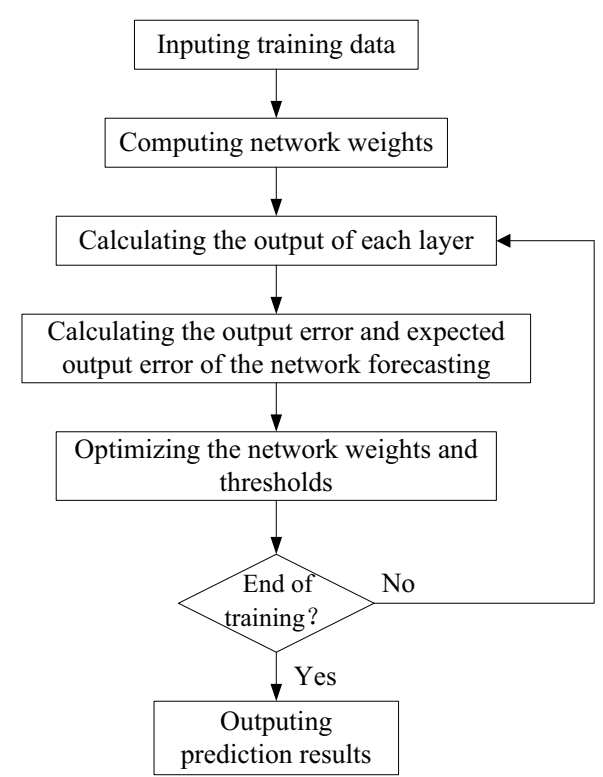

Figure 2 Grey neural network learning process

As shown in the figure: firstly, the network structure and network parameters are initialized according to the characteristic of training data. Then, the weights of network are calculated by the difination and the outputs of A, B, C, D four layers are calculated separately. We obtain the errors between the network forecast output and the expected output. Finally, we get forecast results by optimizing the weights and thresholds according to errors until the end of the training,.

\section{The establishment of grey neural network model of top-oil temperature}

\subsection{The input and output parameters of the network}

The transformer top-oil temperature often depends on three parameters: the first category is environmental parameters, including transformer temperature, humidity, wind speed, solar radiation, etc; the second category is characteristic parameters, mainly refers to transformer winding structure, cooling methods, etc; the third kind is running parameters, such as: load current, active power, reactive power, etc. It is crucial to choose the reasonable parameters for the accurate calculation of transformer top-oil temperature. This paper determines the final characteristic parameters from two aspects: the one is there have a great relevance between the parameters and transformer top-oil temperature; the other one is the network training effect achieves a higher technical indicator by entering the parameters of sample data.

According to the requirements of the two aspects above, the paper determines the environment temperature, load current, active power, reactive power and the top-oil temperature of the previous time as the input parameters of the network.

\subsection{Data acquisition}

Selecting the actual operation data of a transformer substation (the model is the SSZ11-50kV/110 power transformer) of Chengdu in August 2, 2015, we collect the data as a training sample every $5 \mathrm{~min}$ from 00:00 to 23:55. Measuring the temperature of the transformer using optical fiber temperature sensors, the substation provides the historical data of the corresponding environmental temperature, load current, active power, reactive power. In order to meet the requirements of the input of the network, the sample data are normalized and are distributed in the $[0.2,0.8]$ range. As shown in Figure 3-6.

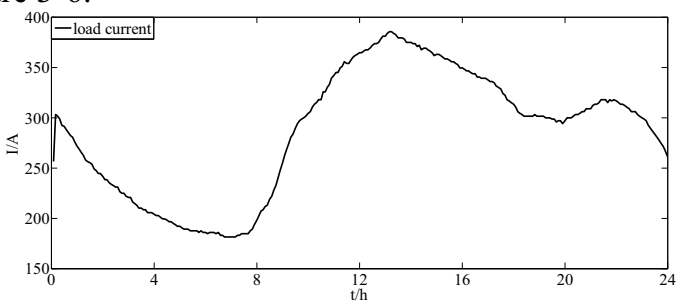

Figure 3 Variation of load current of transformer

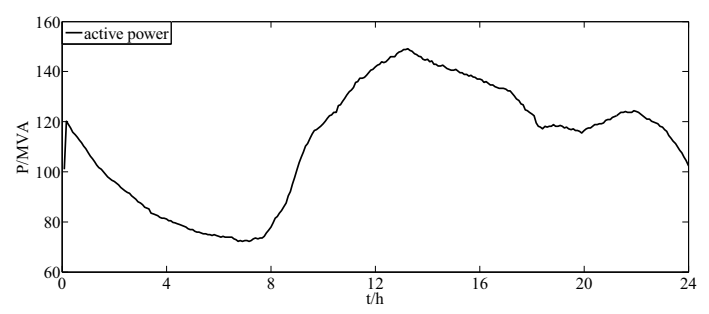

Figure 4 Variation of active power of transformer

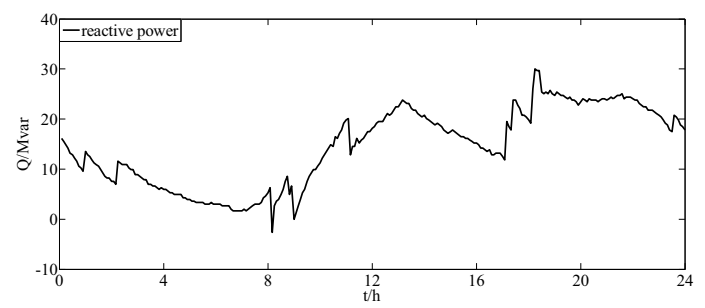

Figure 5 Variation of reactive power of transformer

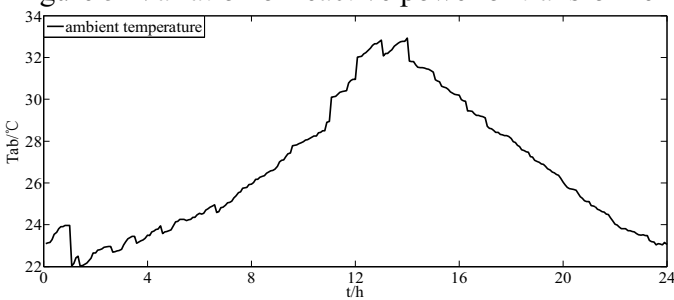

Figure 6 Variation of environment temperature of transformer

\subsection{Training data and test data}

In this paper, the transformer is working under the condition of natural oil circulation. Under the rated load of 24 hours of continuous work, a total of 288 sets of the sample data are measured. The training set and test set are classified according to the 5:1. The 240 groups are finally determined as the training data, and the 48 groups are used as the test data.

\subsection{Network structure design}


The inputs are the environment temperature, load current, active power, reactive power and the top-oil temperature of the previous time. The output is the transformer top-oil temperature. So the structure of the gray neural network is 1-1-5-1.

\subsection{Network training and forecasting}

The forecasting process of transformer top-oil temperature includes three steps based on grey neural network: the construction of grey neural network, the training of grey neural network and the prediction of grey neural network.

First, the impact factors of the transformer top-oil temperature are analyzed, the input and output parameters of the network are determined.

Then, the 240 sets of historical data consisting of a $5 \times 240$ matrix including the environment temperature, load current, active power, reactive power and the top-oil temperature of the previous time are entered. The output is 240 history data of transformer top-oil temperature expressed as a $1 \times 240$ matrix and the grey neural network begins to train.

Finally, we get the desired gray neural network at the end of the training and predict transformer top-oil temperature according to the trained network.

\section{Forecast result and analysis}

Table 1 shows the measured value, BP neural network prediction and grey neural network prediction of 48 groups of top-oil temperature for the test transformer. In order to compare the data visually, the three sets of data are represented in the graph as shown in Figure 7. As can be seen in figure 7, the dynamic changes of two kinds of the prediction models can reflect the changes of top-oil temperature. While compared with the BP network forecasting model, the proposed forecasting model has the better fitting degree and higher accuracy. This shows that the grey neural network model is superior to the BP neural network model in this experiment.

Table 1 Transformer top-oil temperature measured value and

BPNN predictive value and grey network predictive value

\begin{tabular}{|c|c|c|c|}
\hline Time $[\mathrm{min}]$ & $\begin{array}{c}\text { Measured value } \\
{\left[/{ }^{\circ} \mathrm{C}\right]}\end{array}$ & $\begin{array}{c}\text { BPNN } \\
\text { predictive value } \\
{\left[/{ }^{\circ} \mathrm{C}\right]}\end{array}$ & $\begin{array}{c}\text { Grey network } \\
\text { predictive value } \\
{\left[/{ }^{\circ} \mathrm{C}\right]}\end{array}$ \\
\hline 25 & 67.88 & 66.81 & 67.99 \\
\hline 55 & 67.50 & 65.50 & 66.95 \\
\hline 85 & 67.13 & 67.35 & 67.45 \\
\hline 115 & 65.44 & 64.38 & 65.56 \\
\hline 145 & 63.75 & 64.08 & 63.67 \\
\hline 175 & 62.44 & 63.31 & 62.22 \\
\hline 205 & 61.50 & 61.96 & 61.85 \\
\hline 235 & 59.63 & 58.65 & 59.73 \\
\hline 265 & 57.94 & 58.94 & 58.07 \\
\hline 295 & 56.81 & 57.04 & 57.01 \\
\hline 325 & 56.06 & 56.41 & 56.56 \\
\hline 355 & 54.94 & 55.42 & 55.04 \\
\hline 385 & 54.94 & 53.94 & 55.14 \\
\hline 415 & 54.00 & 54.47 & 54.38 \\
\hline 445 & 53.81 & 53.96 & 53.78 \\
\hline 475 & 53.81 & 54.81 & 53.61 \\
\hline 505 & 54.19 & 53.93 & 54.10 \\
\hline 535 & 55.69 & 53.78 & 55.09 \\
\hline 565 & 57.94 & 56.78 & 58.09 \\
\hline 595 & 59.81 & 59.68 & 59.71 \\
\hline & & & \\
\hline
\end{tabular}

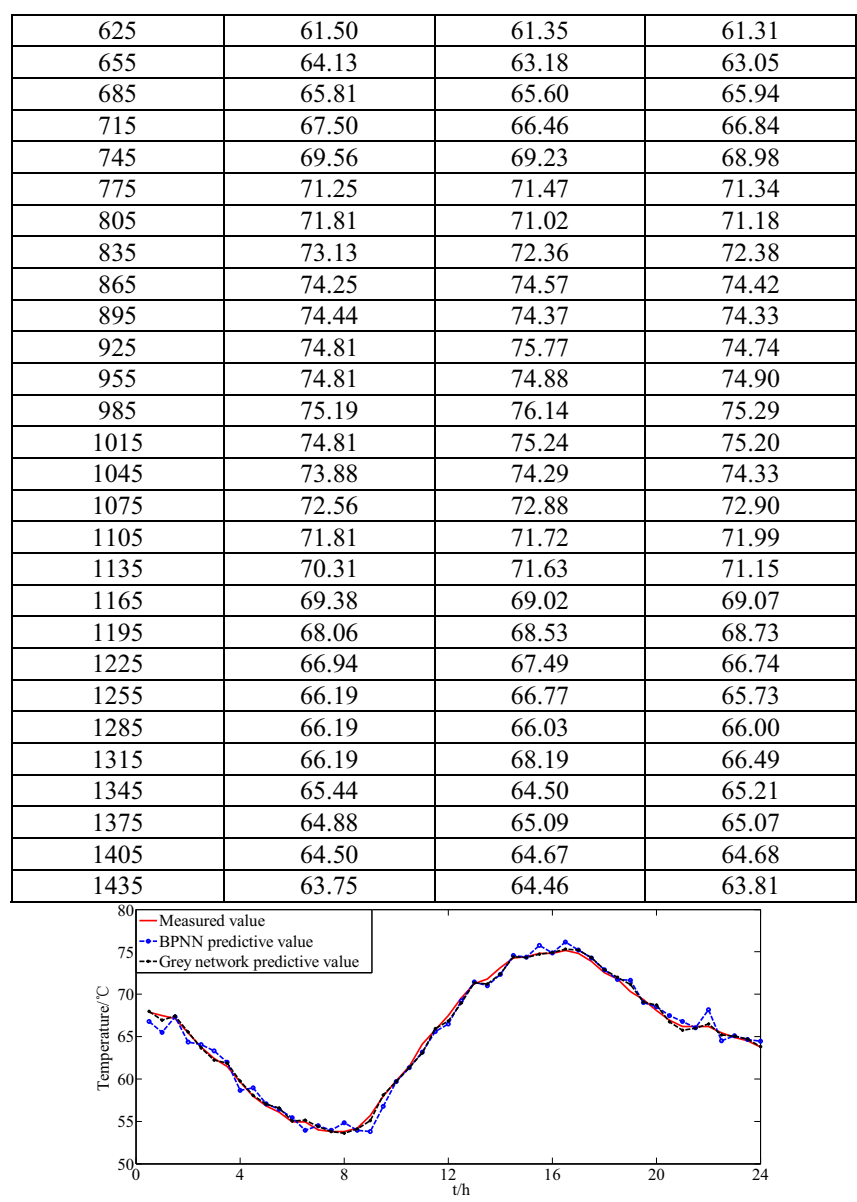

Figure 7 Measured value and prediction value of the top-oil temperature

In order to analyze deeply, a comparative analysis of the relative errors between the BP network model and the grey network model are given by figure 8 and table 2 . From figure 8 , we can see that the relative error of the gray network prediction model is near the zero point and the fluctuation is small. It shows that the relative error is small and stable. As is listed in Table 2, the minimum relative error, average relative error and the maximum relative error of the grey forecasting model are less than those of BP network prediction model. The maximum relative error of the grey forecasting model is $1.68 \%$, which is quite acceptable in engineering.

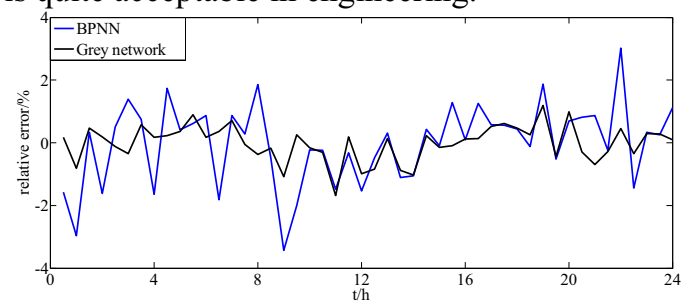

Figure 8 The relative errors between top-oil temperature prediction value and the measured value

Table 2 The relative errors in two model

\begin{tabular}{|c|c|c|c|}
\hline Model & $\delta_{\min }$ & $\bar{\delta}$ & $\delta_{\max }$ \\
\hline BPNN $(\%)$ & 0.10 & 1.03 & 3.44 \\
\hline $\begin{array}{c}\text { Grey } \\
\text { network }(\%)\end{array}$ & 0.05 & 0.47 & 1.68 \\
\hline
\end{tabular}

In the actual operation of the transformer, the environment is complex. In the prediction of top-oil temperature, if we need to consider the wind speed, solar 
radiation, altitude and other factors, and we can directly change the input data of the grey neural network prediction model. For the test transformer, we select the load current, environment temperature, active power, reactive power and the top-oil temperature of the previous time as input parameters.

\section{Conclusion}

The accurate prediction of transformer top-oil temperature is of great significance for the safe operation of power system. Considering the factors affect the transformer top-oil temperature, the paper selects the top oil temperature, load current, active power, reactive power and the top-oil temperature of the previous time as the characteristic parameters, and establishes the prediction model of top-oil temperature based on Grey Neural Network. The applicability, accuracy and feasibility of the model are verified. The example result shows that the prediction model has a fast convergence, good versatility, high precision of the forecasting model which indicats that this is a kind of an effective prediction method of transformer top-oil temperature.

\section{References}

1 Wu Jianjun, Li Xiyuan, Li Sihua, et al. Research on transformer winding fibre optic temperature monitoring system and practical application $[\mathrm{J}]$. Transformer, 50 (11) :47-50 (2013).

2 YANG Honglei, XIONG XIN, LIANG Shibin, et al. Research of depth adjustable FBG oil temperature sensor for transformer[J]. Transducer and Microsystem Technologies, 33 (2) :47-49 (2014).

3 HONG Dan, WEI Guangyuan, QIAN Zheng, et al. Research on application of fiber brag grating sensor in hot-spot temperature measurement for transformer winding $[\mathrm{J}]$. Electrical Measurement \& Instrumentation, 51 (21): 47-51 (2014).

4 IEEE guide for loading mineral-oil-immersed transformers[J]. IEEE guide for loading mineral-oilimmersed transformers, (1996).

5 Lesieutre B C, Hagman W H, Kirtley J L. An improved transformer top oil temperature model for use in an on-line monitoring and diagnostic system[J]. IEEE Transactions on Power Delivery, 1 (12) :249-256 (1997).

6 Tylavsky D J, He Q, Si J, et al. Transformer top-oil temperature modeling and simulation[J]. IEEE Transactions on Industry Applications, 5(36) :12191225 (2000).

7 CHEN Weigen, SU Xiaoping, CHEN Xi, et al. Influence Factor Analysis and Improvement of the Thermal Model for Predicting Transformer Top Oil Temperature[J]. High Voltage Engineering, 37 (6) :1329-1335 (2011).

8 WEI Bengang, HUANG Hua, FU Chenchao, et al. Algorithm for Transformer Top-Oil Temperature and Winding Hot-Point Temperature Based on Modified Thermal Circuit Model[J]. East China
Electric Power, 44 (3) :444-447 (2012).

9 CHEN Jinming, WU Yi, ZHU Haibing, et al. Study and application of Transformer Top-oil predicting $[\mathrm{J}]$. Electrotechnical Application, 22 (33) :89-93 (2015).

10 XIONG Hao, CHEN Weigen, DU Lin, et al. Study on prediction of Top-oil Temperature for Power Transformer Based on T-S Model[J]. Proceedings of the CSEE, 27 (30):15-19(2007).

11 CHEN Weigen, TENG Li, LIU Jun, et al. Transformer winding hot-spot temperature prediction model of support vector machine optimized by genetic algorithm[J]. Transformer of China electrotechnical society, 29 (1) :44-51 (2014).

12 LU Fangcheng, MA Lun, WANG Liu, et al. Calculation of Winding Hot Spot Temperature of Oil Immersed Transformer by the Discontinuous Galerkin Method[J]. High Voltage Apparatus, 51 (3) :28-34 (2015).

13 He Q, Si J N, Tylavsky D J. Prediction of top-oil temperature for transformers using neural networks[J]. IEEE Transactions on Power Delivery, 4(15) :1205-1211 (2000).

14 CHEN Weigen, XI Hongjuan, Su Xiaoping, et al. Application of Generalized Regression Neural Network to Transformer Winding Hot Spot Temperature Forecasting[J]. High Voltage Engineering, 38(1):16-21 (2012).

15 WANG Yongqiang, YUE Guoliang, HE Jie, et al. Study on Prediction of Top Oil Temperature for Power Transformer Based on Kalman Filter Algorithm[J]. High Voltage Apparatus, 50(8):7479 (2014) 